\title{
On the generalized Becker-Stark type inequalities
}

\author{
Yogesh J. Bagul \\ Department of Mathematics, K. K. M. \\ College, Manwath, Dist: \\ Parbhani(M.S.)-431505, India \\ email: yjbagul@gmail.com \\ Christophe Chesneau \\ LMNO, University of Caen-Normandie, \\ Caen, France \\ email: christophe.chesneau@unicaen.fr
}

\author{
Marko Kostić \\ University of Novi Sad, \\ Trg D. Obradovića 6, \\ 21125 Novi Sad, Serbia \\ email: marco.s@verat.net \\ Ramkrishna M. Dhaigude \\ Department of Mathematics, \\ Government Vidarbha Institute of \\ Science and Humanities, \\ Amravati(M. S.)-444604, India \\ email: rmdhaigude@gmail.com
}

\begin{abstract}
In this paper, we establish several generalized Becker-Stark type inequalities for the tangent function. We present unified proofs of many inequalities in the existing literature. Graphical illustrations of some obtained results are also presented.
\end{abstract}

\section{Introduction}

Becker and Stark [6] established the inequality

$$
1-\frac{4 x^{2}}{\pi^{2}}<\frac{x}{\tan x}<\frac{\pi^{2}}{8}-\frac{x^{2}}{2} ; x \in(0, \pi / 2) .
$$

The inequality (1) attracted many researchers and several of its variations and refinements have been established. We may refer to $[8,9,10,20,21,5,11,16$,

2010 Mathematics Subject Classification: 26A48, 26D05, 26D07, 33B10

Key words and phrases: Becker-Stark inequality, Stečkin inequality, tangent function, monotonicity of functions, Bernoulli numbers 
19, 3, 4], and the references therein for more details. Chen and Cheung [8] proved that the best possible constants for which the inequality

$$
\left(1-\frac{4 x^{2}}{\pi^{2}}\right)^{\beta}<\frac{x}{\tan x}<\left(1-\frac{4 x^{2}}{\pi^{2}}\right)^{\alpha} ; x \in(0, \pi / 2)
$$

holds are $\alpha=\pi^{2} / 12 \approx 0.8224$ and $\beta=1$. The inequality (2) refines (1). Recently, Chen and Elezović [9] proved the following inequality:

$$
\frac{\pi^{2}}{12}-\frac{2 x^{3}}{3 \pi}<\frac{x}{\tan x}<1-\frac{8 x^{3}}{\pi^{3}} ; \quad x \in(0, \pi / 2) .
$$

Although the upper bound of (3) is sharper than the corresponding upper bound of (1), it is not sharper than the upper bound in (2).

The inequality

$$
1-\frac{4 x^{2}}{\pi^{2}}<\frac{x}{\tan x}<1-\frac{x^{2}}{3} ; \quad x \in(0, \pi / 2)
$$

was proved by Z.-H. Yang et. al. [19, (96)]. Before we proceed further, we would like to note that the right inequality in (4) is not good near the point $x=\pi / 2-$ as well as that this inequality is not better than the right inequality in (2), as incorrectly stated in [19, Remark 17]. Strictly speaking, the following inequality, which appears as a part of the equation [19, (98)], is not true since the estimate

$$
\left(\frac{\pi^{2}+4 x^{2}}{\pi^{2}-4 x^{2}}\right)^{\pi^{2} / 24}<\frac{3}{3-x^{2}}, \quad x \in(0, \pi / 2)
$$

cannot be satisfied near the point $x=\pi / 2-$. It is also known that

$$
1-\frac{2 x}{\pi}<\frac{x}{\tan x}<\frac{\pi^{2}}{4}-\frac{\pi x}{2} ; x \in(0, \pi / 2) .
$$

The left inequality of (5) is due to H.-F. Ge [12] and the right inequality of (5) is due to S. B. Stečkin [18].

Among all the inequalities (1)-(5), the inequality (2) is the best. In this paper, our aim is to obtain several generalized inequalities by studying the monotonicity of functions with one parameter. We will obtain or refine the above inequalities as particular cases of our results. We also aim to improve the lower bound of $(2)$ in the interval $\left(0, \delta_{*}\right)$ where $\delta_{*} \approx 1.3407$ as well as the upper bound of (2) near the point $x=\pi / 2^{-}$. Our new bounds may not be uniformly better than the ones in (2) but they certainly provide alternatives to the best bounds. 


\section{Preliminaries and lemmas}

The following power series expansions involving Bernoulli numbers can be found in $[13,1.411]$ :

$$
\cot x=\frac{1}{x}-\sum_{k=1}^{\infty} \frac{2^{2 k}}{(2 k) !}\left|B_{2 k}\right| x^{2 k-1} ;|x|<\pi, x \neq 0
$$

and

$$
\csc x=\frac{1}{x}+\sum_{k=1}^{\infty} \frac{2\left(2^{2 k-1}-1\right)}{(2 k) !}\left|B_{2 k}\right| x^{2 k-1} ; \quad|x|<\pi, x \neq 0,
$$

where $B_{2 k}$ are the even indexed Bernoulli numbers. The expansion (7) can be rewritten as

$$
\frac{x}{\sin x}=1+\sum_{\mathrm{k}=1}^{\infty} \frac{2\left(2^{2 \mathrm{k}-1}-1\right)}{(2 \mathrm{k}) !}\left|\mathrm{B}_{2 \mathrm{k}}\right| x^{2 \mathrm{k}} ; \quad|x|<\pi .
$$

From (6), we obtain

$$
\left(\frac{x}{\sin x}\right)^{2}=-x^{2}(\cot x)^{\prime}=1+\sum_{k=1}^{\infty} \frac{(2 k-1) 2^{2 k}}{(2 k) !}\left|B_{2 k}\right| x^{2 k} ; \quad|x|<\pi, \quad x \neq 0 .
$$

Also, with reference to $[13,1.518]$, we have

$$
\ln (\tan x)=\ln x+\sum_{k=1}^{\infty} \frac{\left(2^{2 \mathrm{k}-1}-1\right) 2^{2 \mathrm{k}}}{\mathrm{k}(2 \mathrm{k}) !}\left|\mathrm{B}_{2 \mathrm{k}}\right| x^{2 \mathrm{k}} ; \quad 0<x<\frac{\pi}{2}, \quad x \neq 0 .
$$

In addition to the above formulas, we will also use the following lemmas in order to prove our main results. For Lemma 1, we refer to [2] (see also [7, eqn (4.3), p. 42].

Lemma 1 Let $\mathrm{f}_{1}(\mathrm{x})$ and $\mathrm{f}_{2}(\mathrm{x})$ be two real valued functions which are continuous on $[\mathrm{a}, \mathrm{b}]$ and derivable on $(\mathrm{a}, \mathrm{b})$, where $-\infty<\mathrm{a}<\mathrm{b}<\infty$ and $\mathrm{g}^{\prime}(\mathrm{x}) \neq 0$, for all $x \in(a, b)$. Let,

$$
A(x)=\frac{f_{1}(x)-f_{1}(a)}{f_{2}(x)-f_{2}(a)}, x \in(a, b)
$$

and

$$
B(x)=\frac{f_{1}(x)-f_{1}(b)}{f_{2}(x)-f_{2}(b)}, x \in(a, b) .
$$

Then, we have 
(i) $\mathrm{A}(\mathrm{x})$ and $\mathrm{B}(\mathrm{x})$ are increasing on $(\mathrm{a}, \mathrm{b})$ if $\mathrm{f}_{1}^{\prime}(\mathrm{x}) / \mathrm{f}_{2}^{\prime}(\mathrm{x})$ is increasing on $(a, b)$.

(ii) $\mathrm{A}(\mathrm{x})$ and $\mathrm{B}(\mathrm{x})$ are decreasing on $(\mathrm{a}, \mathrm{b})$ if $\mathrm{f}_{1}^{\prime}(\mathrm{x}) / \mathrm{f}_{2}^{\prime}(\mathrm{x})$ is decreasing on $(a, b)$.

The strictness of the monotonicity of $\mathrm{A}(\mathrm{x})$ and $\mathrm{B}(\mathrm{x})$ depends on the strictness of monotonicity of $\mathrm{f}_{1}^{\prime}(\mathrm{x}) / \mathrm{f}_{2}^{\prime}(\mathrm{x})$.

The result below shows the relationship between two consecutive absolute Bernoulli numbers. It was established recently in [17].

Lemma 2 For $\mathrm{k} \in \mathbb{N}$, the Bernoulli numbers satisfy

$$
\frac{\left(2^{2 k-1}-1\right)}{\left(2^{2 k+1}-1\right)} \frac{(2 k+1)(2 k+2)}{\pi^{2}}<\frac{\left|B_{2 k+2}\right|}{\left|B_{2 k}\right|}<\frac{\left(2^{2 k}-1\right)}{\left(2^{2 k+2}-1\right)} \frac{(2 k+1)(2 k+2)}{\pi^{2}} .
$$

Lemma 3 Let $\mathrm{A}(\mathrm{x})=\sum_{\mathrm{k}=0}^{\infty} \mathrm{a}_{\mathrm{k}} \mathrm{x}^{\mathrm{k}}$ and $\mathrm{B}(\mathrm{x})=\sum_{\mathrm{k}=0}^{\infty} \mathrm{b}_{\mathrm{k}} \mathrm{x}^{\mathrm{k}}$ be convergent for $|x|<R$, where $a_{k}$ and $b_{k}$ are real numbers for $\mathrm{k}=0,1,2, \cdots$ such that $b_{k}>0$ for $\mathrm{k} \geq 0$. If the sequence $\mathrm{a}_{\mathrm{k}} / \mathrm{b}_{\mathrm{k}}$ is strictly increasing (or decreasing), then the function $\mathrm{A}(\mathrm{x}) / \mathrm{B}(\mathrm{x})$ is also strictly increasing (or decreasing) on $(0, \mathrm{R})$.

For more details about Lemma 3, see, for instance, [14]. The following lemma can be found in [1].

Lemma 4 For all integers $\mathrm{k} \in \mathbb{N}$, we have

$$
\frac{2(2 \mathrm{k}) !}{(2 \pi)^{2 \mathrm{k}}} \frac{1}{1-2^{\alpha-2 k}}<\left|\mathrm{B}_{2 \mathrm{k}}\right|<\frac{2(2 \mathrm{k}) !}{(2 \pi)^{2 \mathrm{k}}} \frac{1}{1-2^{\beta-2 k}},
$$

with the best constants $\alpha=0$ and $\beta=2+\left(\ln \left(1-6 / \pi^{2}\right)\right) / \ln 2 \approx 0.6491$.

\section{Main results}

In this section, we will state and prove our main results. In the beginning, for any number $p \in \mathbb{R}$, we define

$$
\phi_{p}(x):=\frac{\tan x-x}{x^{p} \tan x}, \quad x \in(0, \pi / 2) .
$$

Then, the following result holds. 


\section{Theorem 1}

I. $\phi_{p}(x)$ is strictly increasing on $(0, \pi / 2)$ if and only if $\mathrm{p} \leq 2$, and

II. $\phi_{p}(x)$ is strictly decreasing on $(0, \pi / 2)$ if and only if $p \geq \pi^{2} / 4 \approx 2.4674$.

Proof. By differentiation, we have

$$
F_{p}(x)=\tan ^{2} x \cdot \phi_{p}^{\prime}(x)=-p x^{-(p+1)} \tan ^{2} x-(1-p) x^{-p} \tan x+x^{1-p} \sec ^{2} x .
$$

Note that $\phi_{p}(x)$ is strictly increasing on $(0, \pi / 2)$ if and only if $F_{p}(x)>0$, $x \in(0, \pi / 2)$, i.e.,

$$
p<\frac{x^{2} \sec ^{2} x-x \tan x}{\tan x(\tan x-x)}=\frac{\left(\frac{x}{\sin x}\right)^{2}-x \cot x}{1-x \cot x}=: f(x), \quad x \in(0, \pi / 2) .
$$

From (6) and (9), we get

$$
\begin{aligned}
f(x) & =\frac{\sum_{k=1}^{\infty} \frac{2^{2 k}(2 k-1)}{(2 k) !}\left|B_{2 k}\right| x^{2 k}+\sum_{k=1}^{\infty} \frac{2^{2 k}}{(2 k) !}\left|B_{2 k}\right| x^{2 k}}{\sum_{k=1}^{\infty} \frac{2^{2 k}}{(2 k) !}\left|B_{2 k}\right| x^{2 k}} \\
& =\frac{\sum_{k=1}^{\infty} \frac{2^{2 k} 2 k}{(2 k) !}\left|B_{2 k}\right| x^{2 k}}{\sum_{k=1}^{\infty} \frac{2^{2 k}}{(2 k) !}\left|B_{2 k}\right| x^{2 k}}:=\frac{\sum_{k=1}^{\infty} a_{k} x^{2 k}}{\sum_{k=1}^{\infty} b_{k} x^{2 k}}, \quad x \in(0, \pi / 2) .
\end{aligned}
$$

From this, we get $a_{k} / b_{k}=2 k(k \in \mathbb{N})$. Since the sequence $\left\{a_{k} / b_{k}\right\}_{k=1}^{\infty}$ is strictly increasing, we conclude from Lemma 3 that the function $f(x)$ is strictly increasing on $(0, \pi / 2)$. Hence, $\phi_{p}(x)$ is strictly increasing on $(0, \pi / 2)$ if and only if $p \leq \inf \{f(x): 0<x<\pi / 2\}=f\left(0^{+}\right)=2$. Similarly, $\phi_{p}(x)$ is strictly decreasing on $(0, \pi / 2)$ if and only if $F_{p}(x)<0$, which is equivalent to saying that $p \geq \sup \{f(x): 0<x<\pi / 2\}=f\left(\pi / 2^{-}\right)=\pi^{2} / 4$.

Remark 1 Suppose that $\mathrm{p} \in\left(2, \pi^{2} / 4\right)$. Since the function $\mathrm{f}(\mathrm{x})$ is strictly increasing on $(0, \pi / 2)$, we get from the above that there exists a unique point $\mathrm{x}_{\mathrm{p}} \in(0, \pi / 2)$ such that $\mathrm{f}\left(\mathrm{x}_{\mathrm{p}}\right)=\mathrm{p}$. This implies $\mathrm{f}(\mathrm{x})<\mathrm{p}$ for $\mathrm{x} \in\left(0, \mathrm{x}_{\mathrm{p}}\right)$ and $\mathrm{f}(\mathrm{x})>\mathrm{p}$ for $\mathrm{x} \in\left(\mathrm{x}_{\mathrm{p}}, \pi / 2\right)$ so that $\phi_{\mathrm{p}}(\mathrm{x})$ is strictly decreasing on $\left(0, \mathrm{x}_{\mathrm{p}}\right)$ and strictly increasing on $\left(x_{p}, \pi / 2\right)$, with $\phi_{p}(x) \geq \phi_{p}\left(x_{p}\right)$ for $x \in(0, \pi / 2)$.

Let $p \in(-\infty, 4] \backslash\{0\}$. Define now

$$
\psi_{p}(x):=\frac{\ln \left(\frac{x}{\tan x}\right)}{\ln \left(1-p \frac{x^{2}}{\pi^{2}}\right)}, \quad x \in(0, \pi / 2) .
$$

Then, we have: 


\section{Theorem 2}

I. $\psi_{p}(x)$ is strictly decreasing on $(0, \pi / 2)$ if and only if $p<0$, and

II. $\psi_{p}(x)$ is strictly increasing on $(0, \pi / 2)$ if and only if $0<p \leq 4$.

Proof. Set $\psi_{1}(x):=\ln (x / \tan x), x \in(0, \pi / 2)$ and $\left(\psi_{2}\right)_{p}(x):=\ln \left(1-\left(p x^{2} / \pi^{2}\right)\right)$, $x \in(0, \pi / 2)$. Then $\psi_{1}\left(0^{+}\right)=0=\left(\psi_{2}\right)_{p}(0)$ and differentiation yields

$$
\frac{\psi_{1}^{\prime}(x)}{\left(\psi_{2}\right)_{p}^{\prime}(x)}=\frac{1}{2 p}\left(\pi^{2}-p x^{2}\right) \frac{x-\sin x \cos x}{x^{2} \sin x \cos x}=\frac{1}{2 p}\left(\psi_{3}\right)_{p}(x), x \in(0, \pi / 2),
$$

where, for every $x \in(0, \pi / 2)$,

$$
\left(\psi_{3}\right)_{p}(x):=\left(\pi^{2}-p x^{2}\right) \frac{x-\sin x \cos x}{x^{2} \sin x \cos x}=\frac{\left(\pi^{2}-p x^{2}\right)}{x^{2}}\left(\frac{2 x}{\sin 2 x}-1\right) .
$$

By (8), we get

$$
\begin{aligned}
\left(\psi_{3}\right)_{p}(x) & =\frac{\left(\pi^{2}-p x^{2}\right)}{x^{2}} \sum_{k=1}^{\infty} \frac{2^{2 k}-2}{(2 k) !}\left|B_{2 k}\right|(2 x)^{2 k} \\
& =\left(\pi^{2}-p x^{2}\right) \sum_{k=1}^{\infty} \frac{2^{2 k}\left(2^{2 k}-2\right)}{(2 k) !}\left|B_{2 k}\right| x^{2 k-2} \\
& =\pi^{2} \sum_{k=1}^{\infty} \frac{2^{2 k}\left(2^{2 k}-2\right)}{(2 k) !}\left|B_{2 k}\right| x^{2 k-2}-p \sum_{k=1}^{\infty} \frac{2^{2 k}\left(2^{2 k}-2\right)}{(2 k) !}\left|B_{2 k}\right| x^{2 k} \\
& =\frac{2 \pi^{2}}{3}+\sum_{k=1}^{\infty}\left(\frac{\pi^{2} 2^{2 k+2}\left(2^{2 k+2}-2\right)}{(2 k+2) !}\left|B_{2 k+2}\right|-p \frac{2^{2 k}\left(2^{2 k}-2\right)}{(2 k) !}\left|B_{2 k}\right|\right) x^{2 k} \\
& =\frac{2 \pi^{2}}{3}+\sum_{k=1}^{\infty} a_{k} x^{2 k}, \quad x \in(0, \pi / 2),
\end{aligned}
$$

where

$$
a_{k}:=\frac{\pi^{2} 2^{2 k+2}\left(2^{2 k+2}-2\right)}{(2 k+2) !}\left|B_{2 k+2}\right|-p \frac{2^{2 k}\left(2^{2 k}-2\right)}{(2 k) !}\left|B_{2 k}\right| \quad(k \in \mathbb{N}) .
$$

Case I. If $p<0$, then $a_{k}>0$ for $k \in \mathbb{N}$ and $\left(\psi_{3}\right)_{p}(x)$ is strictly increasing on $(0, \pi / 2)$. Consequently, $\psi_{p}(x)$ is strictly decreasing on $(0, \pi / 2)$ by Lemma 1. 
Case II. If $p \in(0,4]$, then $-p \geq-4$ and we have

$$
a_{k} \geq \frac{\pi^{2} 2^{2 k+2}\left(2^{2 k+2}-2\right)}{(2 k+2) !}\left|B_{2 k+2}\right|-4 \frac{2^{2 k}\left(2^{2 k}-2\right)}{(2 k) !}\left|B_{2 k}\right|=: j(k), \quad k \in \mathbb{N} .
$$

From Lemma 4, we calculate

$$
\begin{aligned}
a_{k} \geq j(k) & >\frac{2^{2 k+3}}{\pi^{2 k}}\left(\frac{2^{2 k+2}-2}{2^{2 k+2}-1}-\frac{2^{2 k}-2}{2^{2 k}-2^{\beta}}\right) \\
& =\frac{2^{2 k+3}}{\pi^{2 k}} \cdot \frac{2^{2 k+2}\left(2-2^{\beta}\right)-2^{2 k}}{\left(2^{2 k+2}-1\right)\left(2^{2 k}-2^{\beta}\right)} \\
& =\frac{2^{4 k+3}}{\pi^{2 k}} \cdot \frac{4\left(2-2^{\beta}\right)-1}{\left(2^{2 k+2}-1\right)\left(2^{2 k}-2^{\beta}\right)} .
\end{aligned}
$$

Since $4\left(2-2^{\beta}\right) \approx 1.7268$, we get $a_{k}>0$ for $k \in \mathbb{N}$. This shows that $\left(\psi_{3}\right)_{p}(x)$ is strictly increasing on $(0, \pi / 2)$. By Lemma $1, \psi_{p}(x)$ is also strictly increasing on $(0, \pi / 2)$.

If $p<4$, then the function $\psi_{p}(x)$ cannot be defined for $x \geq \pi / 2$. Suppose now that $p>4$ and consider the function $\psi_{p}(x)$ defined for $x \in(0, \pi / \sqrt{p})$. Then, the following result holds.

Theorem 3 The function $\psi_{\mathrm{p}}(\mathrm{x})$ is strictly decreasing on $(0, \pi / \sqrt{\mathrm{p}})$ if $\mathrm{p} \geq$ $84 / 15$.

Proof. Repeating verbatim the arguments used in the proof of Theorem 2, we get that

$$
\left(\psi_{3}\right)_{p}(x)=\frac{2 \pi^{2}}{3}+\sum_{k=1}^{\infty} a_{k} x^{2 k}, \quad x \in(0, \pi / 2),
$$

where $a_{k}(k \in \mathbb{N})$ is given through (12). Let $c:=84 /\left(15 \pi^{2}\right)$. Then, we have

$$
a_{k} \leq \pi^{2}\left(\frac{2^{2 k+2}\left(2^{2 k+2}-2\right)}{(2 k+2) !}\left|B_{2 k+2}\right|-c \frac{2^{2 k}\left(2^{2 k}-2\right)}{(2 k) !}\left|B_{2 k}\right|\right)=: l(k), \quad k \in \mathbb{N} .
$$

Then $l(k)<0$ if and only if

$$
\frac{\left|B_{2 k+2}\right|}{\left|B_{2 k}\right|}<c \frac{2^{2 k}\left(2^{2 k}-2\right)}{(2 k) !} \frac{(2 k+2) !}{2^{2 k+2}\left(2^{2 k+2}-2\right)}
$$




$$
=\frac{c}{4} \frac{(2 k+1)(2 k+2)\left(2^{2 k-1}-1\right)}{\left(2^{2 k+1}-1\right)} .
$$

From Lemma 2, we have

$$
\frac{\left|B_{2 k+2}\right|}{\left|B_{2 k}\right|}<\frac{\left(2^{2 k}-1\right)}{\left(2^{2 k+2}-1\right)} \frac{(2 k+1)(2 k+2)}{\pi^{2}} .
$$

Keeping in mind the arguments used in the proof of Theorem 2, it remains to be shown that

$$
4\left(2^{2 k}-1\right)\left(2^{2 k+1}-1\right)<c \pi^{2}\left(2^{2 k-1}-1\right)\left(2^{2 k+2}-1\right), k \in \mathbb{N} .
$$

After making a substitution $x=4^{k}(x \geq 4)$, it suffices to show that

$$
2 \frac{(x-1)(2 x-1)}{(x-2)(4 x-1)} \leq \frac{c}{4} \pi^{2}, \quad x \geq 4 .
$$

The equality holds for $x=4$, while the strict inequality holds for $x>4$ because the function

$$
y=2 \frac{(x-1)(2 x-1)}{(x-2)(4 x-1)}, \quad x \geq 4
$$

is strictly decreasing (https://www.desmos.com/calculator), as it can be easily approved.

Next, we will show how our results give some known and other inequalities for $x / \tan x$. First of all, we can see by Theorem 1 that the function

$$
\phi_{2}(x)=\frac{\tan x-x}{x^{2} \tan x}
$$

is strictly increasing on $(0, \pi / 2)$. Hence,

$$
\phi_{2}\left(0^{+}\right)=\frac{1}{3}<\phi_{2}(x)=\frac{\tan x-x}{x^{2} \tan x}<\phi_{2}\left(\pi / 2^{-}\right)=\frac{4}{\pi^{2}},
$$

which gives the inequality (4). Similarly, $\phi_{1}(x)$ is strictly increasing on $(0, \pi / 2)$ and thus with limits at extremities we obtain

$$
1-\frac{2 x}{\pi}<\frac{x}{\tan x}<1 ; \quad x \in(0, \pi / 2) .
$$

This gives the left inequality of (5). Looking at the strictly decreasing function $\phi_{3}(x)$ on $(0, \pi / 2)$ and the limit $\phi_{3}\left(\pi / 2^{-}\right)=8 / \pi^{3}$, we get the right inequality 
of (3). Indeed, this inequality can be sharpened by considering $\phi_{p}(x)$ for $p=$ $\pi^{2} / 4$. Since $\left(\phi_{p}(x)\right)_{p=\pi^{2} / 4}$ is strictly decreasing on $(0, \pi / 2)$, we obtain

$$
\left(\phi_{\mathfrak{p}}(x)\right)_{p=\pi^{2} / 4}>\left(\phi_{\mathfrak{p}}\left(\pi / 2^{-}\right)\right)_{p=\pi^{2} / 4},
$$

i.e.,

$$
\frac{x}{\tan x}<1-\left(\frac{2 x}{\pi}\right)^{\pi^{2} / 4} ; x \in(0, \pi / 2) .
$$

The inequality (14) is better than the right inequality of (2) near the point $x=\pi / 2^{-}$. However, there is no strict comparison between the two.

Now it is easy to formulate the following

Corollary 1 The exponents 2 and $\pi^{2} / 4$ such that

$$
1-\left(\frac{2 x}{\pi}\right)^{2}<\frac{x}{\tan x}<1-\left(\frac{2 x}{\pi}\right)^{\pi^{2} / 4} ; x \in(0, \pi / 2)
$$

are optimal.

Proof. Let

$$
g(x)=\frac{\ln \left(1-\frac{x}{\tan x}\right)}{\ln \left(\frac{2 x}{\pi}\right)}=\frac{g_{1}(x)}{g_{2}(x)} .
$$

Here $g_{1}(x)$ and $g_{2}(x)$ are such that $g_{1}\left(\pi / 2^{-}\right)=0=g_{2}(\pi / 2)$. Then

$$
\frac{g_{1}^{\prime}(x)}{g_{2}^{\prime}(x)}=\frac{x^{2} \sec ^{2} x-x \tan x}{\tan x \cdot(\tan x-x)}=f(x)
$$

which is strictly increasing on $(0, \pi / 2)$ as discussed in the proof of Theorem 1 . Calculating the limits at extremities, we obtain the required.

Several other inequalities can be established by using Theorem 1. We also have the following corollaries of Theorem 2 .

Corollary 2 If $p \in(0,4]$ and $x \in(0, \lambda)$, where $\lambda \in(0, \pi / 2]$, then the inequalities

$$
\left(1-p \frac{x^{2}}{\pi^{2}}\right)^{\alpha}<\frac{x}{\tan x}<\left(1-p \frac{x^{2}}{\pi^{2}}\right)^{\beta}
$$

hold with the best possible constants $\alpha=\psi_{p}\left(\lambda^{-}\right)$and $\beta=\pi^{2} / 3 p$. 
Proof. From Theorem 2, $\psi_{p}(x)$ is strictly increasing on $(0, \lambda)$ for $p \in(0,4]$. So,

$$
\psi_{p}\left(0^{+}\right)<\psi_{p}(x)<\psi\left(\lambda^{-}\right) .
$$

Since $\psi_{p}\left(0^{+}\right)=\pi^{2} / 3 p$, we get $(16)$.

Remark 2 The inequality (2) can be deduced from Corollary 2, with $p=4$ and $\lambda=\pi / 2$.

Corollary 3 If $\mathrm{a}>0$, then the following inequality holds:

$$
\frac{x}{\tan x}<\left(\frac{\pi^{2}}{\pi^{2}+a x^{2}}\right)^{\pi^{2} / 3 a} ; x \in(0, \pi / 2) .
$$

Remark 3 Graphically it is observed that the inequality (17) is in fact true for $x \in(0, \pi)$.

We can use Theorem 3 to prove the following important corollary:

Corollary 4 If $x \in(0, \pi / \sqrt{p})$, where $p \geq 84 / 15$, then the following inequality holds:

$$
\frac{x}{\tan x} \geq\left(1-p \frac{x^{2}}{\pi^{2}}\right)^{\pi^{2} / 3 p} .
$$

Furthermore, $\alpha=\pi^{2} / 3 p$ is the optimal value for which (18) holds with a number $\mathrm{p} \geq 84 / 15$ given in advance.

Albeit not used henceforward, we will state and prove the following result:

Proposition 1 Suppose that $0<p_{1}<p_{2}$ and $x \in\left(0, \pi / \sqrt{p_{2}}\right)$. Then, we have

$$
\left(1-p_{2} \frac{x^{2}}{\pi^{2}}\right)^{\pi^{2} / 3 p_{2}}<\left(1-p_{1} \frac{x^{2}}{\pi^{2}}\right)^{\pi^{2} / 3 p_{1}} .
$$

Proof. Let $0<a<1$. Then, the mapping $t \mapsto \ln (1-a t)-a \ln (1-t)$, $0 \leq t<1$ is strictly increasing because its first derivative is given by

$$
t \mapsto a(1-a) t(1-t)^{-1}(1-a t)^{-1}, \quad t \in[0,1) .
$$

Therefore, we have

$$
\ln (1-a t)>a \ln (1-t), \quad 0<a<1,0<t<1 .
$$


Applying (20) with $a=p_{1} / p_{2}$ and $t=p_{2} x^{2} / \pi^{2}$, we get

$$
\frac{\ln \left(1-p_{2} x^{2} / \pi^{2}\right)}{p_{2}}<\frac{\ln \left(1-p_{1} x^{2} / \pi^{2}\right)}{p_{1}} .
$$

Multiplying both sides of the above inequality with $\pi^{2} / 3$ and taking the exponents, we immediately get (19).

Suppose now that $4<p<84 / 15$. We want to better explore the inequality (18) and the right part of the inequality (2) in this intermediate case. First of all, it is clear that there exists a sufficiently small real number $\epsilon_{\mathrm{p}}>0$ such that

$$
\frac{x}{\tan x}>\left(1-p \frac{x^{2}}{\pi^{2}}\right)^{\pi^{2} / 3 p}, \quad x \in\left((\pi / \sqrt{p})-\epsilon_{p}, \pi / \sqrt{p}\right) .
$$

Set now

$$
A:=\left\{p>4 ; \frac{x}{\tan x}>\left(1-p \frac{x^{2}}{\pi^{2}}\right)^{\pi^{2} / 3 p} \text { for all } x \in(0, \pi / \sqrt{p})\right\} .
$$

By Corollary 4 , we have $[84 / 15,+\infty) \subseteq A$. On the other hand, Proposition yields that, if $p_{0}>4$ and $p_{0} \notin A$, then $\left(4, p_{0}\right] \cap A=\emptyset$. Therefore, it is natural to ask: Can we calculate the set $A$ intrinsically?

The answer is affirmative as the next result shows:

Theorem 4 We have $\mathrm{A}=\left[7 \pi^{2} / 15,+\infty\right)$.

Proof. Define

$$
h(x):=\ln \left(\frac{x}{\tan x}\right)-\frac{\pi^{2}}{3 p} \ln \left(1-p \frac{x^{2}}{\pi^{2}}\right), \quad x \in(0, \pi / \sqrt{p}) .
$$

Then $h(0+)=0$ and

$$
\begin{aligned}
h^{\prime}(x) & =\frac{3 \pi^{2}+x^{2}\left(2 \pi^{2}-3 p\right)}{3 x\left(\pi^{2}-p x^{2}\right)}-\frac{2}{\sin (2 x)} \\
& =\frac{\left[3 \pi^{2}+x^{2}\left(2 \pi^{2}-3 p\right)\right] \sin (2 x)-6 x\left(\pi^{2}-p x^{2}\right)}{3 x\left(\pi^{2}-p x^{2}\right) \sin (2 x)}, \quad x \in(0, \pi / \sqrt{p}) .
\end{aligned}
$$

Set $t:=2 x \in(0,2 \pi / \sqrt{p})$ and

$$
g(t):=\frac{\sin t}{t}-\frac{12 \pi^{2}-3 p t^{2}}{12 \pi^{2}+t^{2}\left(2 \pi^{2}-3 p\right)}, \quad t \in(0,2 \pi / \sqrt{p}) .
$$


Then, it can be easily seen that $h^{\prime}(x)>0$ if and only if $g(t)>0$ if and only if $q(t)>0$, where

$$
q(t):=\sin (t) \cdot\left[12 \pi^{2}+t^{2}\left(2 \pi^{2}-3 p\right)\right]-t\left[12 \pi^{2}-3 p t^{2}\right], \quad t \in[0,2 \pi / \sqrt{p}) .
$$

Using https://www.symbolab.com/solver/partial-derivative-calculator, we get that $\mathrm{q}^{(\mathrm{i})}(0)=0$ for $i=0,1,2,3,4$ as well as that

$$
q^{(v)}(t)=t^{2} \cos t \cdot\left(2 \pi^{2}-3 p\right)+10 t \sin t \cdot\left(2 \pi^{2}-3 p\right)+\cos t \cdot\left(60 p-28 \pi^{2}\right),
$$

for any $t \in[0,2 \pi / \sqrt{p})$. Since $2 \pi^{2}-3 p>0$ for $p<84 / 15$, we have that the assumption $p \geq 7 \pi^{2} / 15$ implies $q^{(v)}(0) \geq 0$ and $q^{(v)}(t)>0$ for all $t \in$ $(0,2 \pi / \sqrt{p})$. This simply implies $q(t)>0$ for all $t \in(0,2 \pi / \sqrt{p})$ and therefore the function $h(x)$ is strictly increasing on $(0, \pi / \sqrt{p})$; therefore $h(x)>h(0+)=$ 0 for all $x \in(0, \pi / \sqrt{p})$ and $\left[7 \pi^{2} / 15,+\infty\right) \subseteq A$. If $p<7 \pi^{2} / 15$, then we have $q^{(i)}(0)=0$ for $i=0,1,2,3,4$ and $q^{(v)}(0)<0$, so that $t=0$ is a local maximum of function $q(t)$ (which can be extended to the even function defined on the whole real line) and therefore $\mathrm{q}(\mathrm{t})<0$ in a right neighborhood of point $t=0$, which implies that $h^{\prime}(x)<0$ in a right neighborhood of point $x=0$ and therefore $h(x)<0$ in a right neighborhood of point $x=0$; hence, $p \notin A$. Theorem 4 is proved.

We now propose an alternative proof of Theorem 4 through the same baseline and the use of power series expansions.

Proof. [Alternative proof] Define

$$
h(x):=\ln \left(\frac{x}{\tan x}\right)-\frac{\pi^{2}}{3 p} \ln \left(1-p \frac{x^{2}}{\pi^{2}}\right), \quad x \in(0, \pi / \sqrt{p}) .
$$

Then, by the power series expansion of the logarithmic function and (10), we have

$$
h(x)=\frac{\pi^{2}}{3 p} \sum_{k=1}^{\infty} \frac{1}{k} \frac{p^{k}}{\pi^{2 k}} x^{2 k}-\sum_{k=1}^{\infty} \frac{\left(2^{2 k-1}-1\right) 2^{2 k}}{k(2 k) !}\left|B_{2 k}\right| x^{2 k} .
$$

Since $\left|B_{2}\right|=1 / 6$, after simplification, we get

$$
h(x)=\sum_{k=2}^{\infty} c_{2 k} x^{2 k}
$$

where

$$
c_{2 k}:=\frac{1}{k}\left[\frac{1}{3} \frac{p^{k-1}}{\pi^{2 k-2}}-\frac{\left(2^{2 k-1}-1\right) 2^{2 k}}{(2 k) !}\left|B_{2 k}\right|\right] .
$$


Now, since $\left|B_{4}\right|=1 / 30$, we have $c_{4}=p /\left(6 \pi^{2}\right)-7 / 90$. So, if $p \geq 7 \pi^{2} / 15$, we obtain $c_{4} \geq 0$. The rest of the proof consists in proving that $c_{2 k}>0$ for $k \geq 3$. It follows from Lemma 4 that, for any $k \in \mathbb{N}$,

$$
\left|\mathrm{B}_{2 \mathrm{k}}\right|<\frac{2^{2 \mathrm{k}-1}}{2^{2 \mathrm{k}-1}-1} \frac{2(2 \mathrm{k}) !}{(2 \pi)^{2 \mathrm{k}}}
$$

which implies that

$$
\frac{\left(2^{2 k-1}-1\right) 2^{2 k}}{(2 k) !}\left|B_{2 k}\right|<\frac{2^{4 k}}{(2 \pi)^{2 k}}=\left(\frac{4}{\pi^{2}}\right)^{k}
$$

Therefore, if $p \geq 7 \pi^{2} / 15$, the following inequality holds:

$$
c_{2 k}>\frac{1}{k}\left[\frac{1}{3}\left(\frac{7}{15}\right)^{k-1}-\left(\frac{4}{\pi^{2}}\right)^{k}\right] .
$$

Now, remark that the inequality $(1 / 3)(7 / 15)^{k-1}>\left(4 / \pi^{2}\right)^{k}$ is equivalent to $21 / 15<\left(7 \pi^{2} / 60\right)^{k}$, which is true for $k \geq 3$ since $21 / 15=1.4,7 \pi^{2} / 60 \approx$ $1.151454>1$ and $\left(7 \pi^{2} / 60\right)^{3} \approx 1.52665$. Thus, for $k \geq 3$, we have $c_{2 k}>0$. Now, if $p<7 \pi^{2} / 15$, we have $c_{4}<0$. Owing to the expansion (22) and [15], there exists a $\delta>0$ such that $h(x)<0$ for $x \in(0, \delta)$. This ends the proof of Theorem 4.

Now, Corollary 4 holds with $p \geq 7 \pi^{2} / 15$. For $p=7 \pi^{2} / 15$, from Corollary 4, we get

$$
\left(1-\frac{7 x^{2}}{15}\right)^{15 / 21}<\frac{x}{\tan x} ; \quad x \in(0, \delta),
$$

where $\delta=\sqrt{15 / 7} \approx 1.46385 \cdots$.

Now, let us compare graphically the bounds of $x / \tan x$ given in (2) with those obtained in (14) and in (23) in Figures 1 and 2, respectively. In each case, we distinguish two non-overlapping intervals of values for $x$ to show some hierarchy for these bounds.

Based on Figure 1 and a numerical analysis, we see that, for $x \in\left(0, \delta_{*}\right)$ where $\delta_{*} \approx 1.3407$, the lower bound in (23) is stronger than the lower bound in $(2)$. It is weaker for $x \in\left(\delta_{*}, \delta\right)$, where $\delta \approx 1.4638$. Also, based on Figure 2 and a numerical analysis, for $x \in(0, \mu)$, where $\mu \approx 1.1913$, the upper bound in (2) is stronger than the upper bound in (14). It is weaker for $x \in(\mu, \pi / 2)$. 


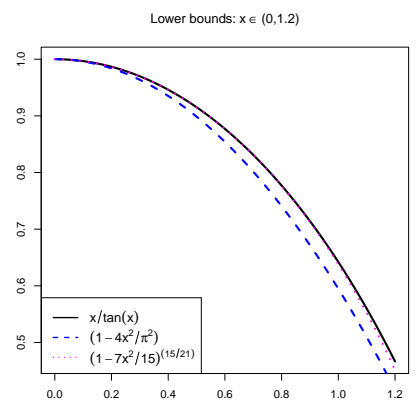

(a)

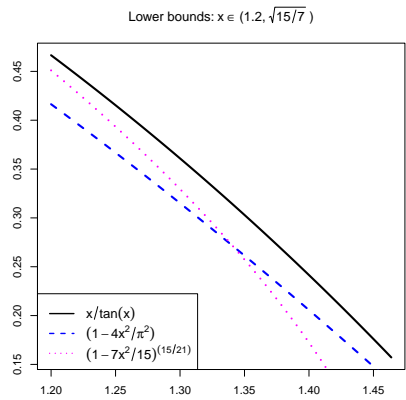

(b)

Figure 1: Graphs of lower bounds of $x / \tan x$ in $(2)$ and (23) for $(a) x \in(0,1.2)$ and $(b) x \in(1.2, \sqrt{15 / 7})$.

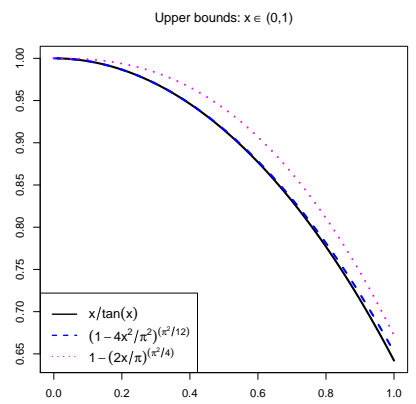

(a)

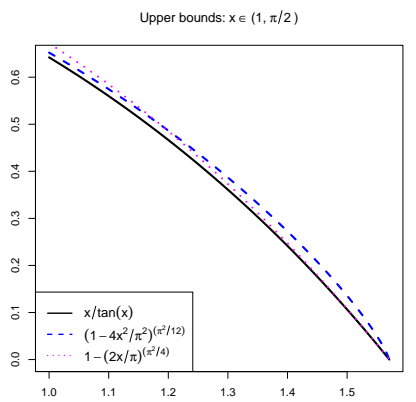

(b)

Figure 2: Graphs of upper bounds of $x / \tan x$ in $(2)$ and (14) for $(a) x \in(0,1)$ and (b) $x \in(1, \pi / 2)$.

We conclude the paper by posing an open problem as follows:

Open Problem. Suppose that $p, \zeta>0$. Then, determine the best possible constants $\alpha_{\mathfrak{p}, \zeta}, \beta_{\mathfrak{p}, \zeta} \in \mathbb{R}$ such that the inequality

$$
\left(1-\frac{p x^{\zeta}}{\pi^{\zeta}}\right)^{\beta_{p, \zeta}}<\frac{x}{\tan x}<\left(1-\frac{p x^{\zeta}}{\pi^{\zeta}}\right)^{\alpha_{p}, \zeta} ; x \in(0, \pi / 2) \cap\left(0, \pi / p^{1 / \zeta}\right)
$$

holds. 


\section{Declarations}

The authors declare no conflict of interest. The authors declare no funding. All the authors contributed equally to the findings.

\section{Acknowledgments}

The authors would like to warmly thank Professor Branko Malešević for thorough comments, constructive discussions and the elegant alternative proof of Theorem 4 .

The authors are very grateful to the referee for improving the article with constructive comments.

\section{References}

[1] H. Alzer, Sharp bounds for the Bernoulli numbers, Archiv der Mathematik, Vol. 74, No. 3 (2000), 207-211.

[2] G. D. Anderson, M. K. Vamanamurthy and M. Vuorinen, Conformal Invariants, Inequalities and Quasiconformal maps, John Wiley and Sons, New York, 1997.

[3] Y. J. Bagul, On simple Jordan type inequalities, Turkish J. Ineq., Vol. 3, No. 1 (2019), 1-6.

[4] Y. J. Bagul, and C. Chesneau, New sharp bounds for tangent function, Bulletin of the Allahabad Mathematical Society, Vol. 34, No. 2 (2019), 277-282.

[5] B. Banjac, M. Makragić and B. Malešević, Some notes on a method for proving inequalities by computer, Results in Mathematics, Vol. 69, No. 1 (2016), 161-176.

[6] M. Becker, and E. L. Stark, On a hierarchy of quolynomial inequalities for tan x, Univ., Beograd. Publ. Elektrotehn. Fak. Ser. Mat. Fiz., No. 602/633 (1978), 133-138.

[7] J. Cheeger, M. Gromov, M. Taylor, Finite propagation speed, kernel estimates for functions of the Laplace operator, and the geometry of complete Riemannian manifolds, J. Differential Geom., Vol. 17, No. 1 (1982), 1553. 
[8] C.-P. Chen, and W.-S.Cheung, Sharp Cusa and Becker-Stark inequalities, J. Inequal. Appl., 2011:136 (2011), Doi: https://doi.org/10.1186/1029242X-2011-136.

[9] C.-P. Chen, and N. Elezović, Sharp Redheffer-type and Becker-Stark-type inequalities with an application, Mathematical Inequalities and Applications, Vol. 21, No. 4 (2018), 1059-1078.

[10] C.-P. Chen, and J. Sándor, Sharp inequalities for trigonometric and hyperbolic functions, Journal of Mathematical Inequalities, Vol. 9, No. 1 (2015), 203-217.

[11] L. Debnath, C. Mortici and L. Zhu, Refinements of Jordan-Stečkin and Becker-Stark inequalities, Results. Math. Vol. 67 (2015), 207-215.

[12] H.-F. Ge, New sharp bounds for the Bernoulli numbers and refinement of Becker-Stark inequalities, J. Inequal. Appl., (2012), Article ID 137507, 7 pages.

[13] I. S. Gradshteyn and I. M. Ryzhik, Table of Integrals, Series and Products, Elsevier, Seventh Edition, 2007.

[14] V. Heikkala, M. K. Vamanamurthy and M. Vuorinen, Generalized elliptic integrals, Comput. Methods Funct. Theory, Vol. 9, No. 1 (2009), 75-109.

[15] B. Malešević and M. Makragić, Method for Proving Some Inequalities on Mixed Trigonometric Polynomial Functions, J. Math. Inequal., Vol. 10, No. 3 (2016), 849-876.

[16] Y. Nishizawa, Sharp Becker-Stark's type inequalities with power exponential functions, J. Inequal. Appl., 2015, 402.

[17] F. Qi, A double inequality for the ratio of two non-zero neighbouring Bernoulli numbers, Journal of Computational and Applied Mathematics, Vol. 351, 1 May (2019), 1-5.

[18] S. B. Stečkin, Some remarks on trigonometric polynomials, Uspekhi Matematicheskikh Nauk, Vol. 10, No. 1 (63) (1955), 159-166 (in Russian).

[19] Z.-H. Yang, Y.-L. Jiang, Y.-Q. Song and Y.-M. Chu, Sharp inequalities for trigonometric functions, Abstract and Applied Analysis, Vol. 2014, Article ID 601839 (2014), 18 pages. 
[20] L. Zhu, Simple proofs of the Cusa-Huygens-type and Becker-Stark-type inequalities, Journal of Mathematical Inequalities, Vol. 7, No. 4 (2013), $563-567$.

[21] L. Zhu, and J.-K. Hua, Sharpening the Becker-Stark inequalities, J. Inequal. Appl., Article ID 931275 (2010). 